\title{
The Laplace-Adomian-Pade Technique for the ENSO Model
}

\author{
Yi Zeng \\ Key Laboratory of Numerical Simulation of Sichuan Province and College of Mathematics and Information Science, \\ Neijiang Normal University, Neijiang 641100, China \\ Correspondence should be addressed to Yi Zeng; mathzy@126.com
}

Received 28 December 2012; Accepted 21 January 2013

Academic Editor: H. Jafari

Copyright @ 2013 Yi Zeng. This is an open access article distributed under the Creative Commons Attribution License, which permits unrestricted use, distribution, and reproduction in any medium, provided the original work is properly cited.

The Laplace-Adomian-Pade method is used to find approximate solutions of differential equations with initial conditions. The oscillation model of the ENSO is an important nonlinear differential equation which is solved analytically in this study. Compared with the exact solution from other decomposition methods, the approximate solution shows the method's high accuracy with symbolic computation.

\section{Introduction}

In recent years, El Niño/La Niña-Southern Oscillation (ENSO) is a quasiperiodic climate pattern that occurs across the tropical Pacific Ocean every five years which has caught more and more attention of researchers due to its great destructions. It is coupled with two phases, the warm oceanic phase, El Niño, and the cold phase, La Niña. Some methods were applied to consider the numerical simulation, among which is the famous Adomian decomposition method (ADOM) [1].

Generally speaking, two aspects affect the accuracy of the ADOM: the calculation of the Adomian decomposition series and the initial iteration value. In view of these points, various modified versions are proposed to solve the nonlinear initial value problems [2-7].

Recently, Tsai and Chen [8-10] suggested a LaplaceAdomian-Pade method (LAPM) to approximately solve the initial value problems of differential equations. The method holds the following merits: (a) the Laplace transformation can be used to "fully" determine the initial iteration value; (b) the Adomian series is used to linearize the nonlinear terms; (c) the Pade technique is used to accelerate the convergence and enlarge the valid area of the approximate solution.

In this paper, we use the method to approximately solve the ENSO model. The approximate solution is compared with other nonlinear techniques in the high order iteration and the result shows the method's higher accuracy.

\section{Approximate Solutions of the ENSO Model}

The air-sea coupled dynamical system was used to describe the oscillating physical mechanism of the ENSO [11]

$$
\begin{gathered}
\frac{d T}{d t}=C T+D h-\varepsilon T^{3}, \\
\frac{d h}{d t}=-E T-R_{h} h,
\end{gathered}
$$

where $C, D, E$, and $R_{h}$ are physical constants, $T$ describes the temperature of the eastern equatorial Pacific sea surface, and $h$ is the thermo-cline depth anomaly. The model (1) shows the variations of both eastern and western Pacific anomaly patterns.

Case I. When $D=0$ and $0<\varepsilon \ll 1$, then (1) can be reduced to

$$
\frac{d T}{d t}=C T-\varepsilon T^{3}
$$

In order to solve (2) with the LAPM, apply the Laplace transform $L$ to both sided of (2) first and we can derive

$$
s \bar{T}(s)=T(0)+C T(s)-\varepsilon L\left[T^{3}\right],
$$

where $\bar{T}(s)=\int_{0}^{\infty} e^{-s t} T(t) d t$. As a result, (3) leads to

$$
\bar{T}(s)=\frac{T(0)}{(s-C)}-\frac{\varepsilon L\left[T^{3}\right]}{(s-C)} .
$$


Apply the inverse of the Laplace transform $L^{-1}$ and expand the nonlinear term as an Adomian series $[1,12]$; then (4) can be written as

$$
\sum_{n=0}^{\infty} v_{n}(t)=L^{-1}\left[\frac{T(0)}{(s-C)}\right]-L^{-1}\left[\frac{\varepsilon L\left[\sum_{n=0}^{\infty} A_{n}\right]}{(s-C)}\right],
$$

where $T(t)=\sum_{n=0}^{\infty} v_{n}(t)$ and $\sum_{n=0}^{\infty} A_{n}$ is the Adomian series of $T^{3}$; namely,

$$
\begin{gathered}
A_{0}=v_{0}^{3}, \\
A_{1}=3 v_{0}^{2} v_{1}, \\
A_{2}=3 v_{0} v_{1}^{2}+3 v_{0}^{2} v_{2},
\end{gathered}
$$

Now the iteration formula can be determined for (2) as

$$
\begin{gathered}
v_{n+1}(t)=-L^{-1}\left[\frac{\varepsilon L\left[A_{n}\right]}{(s-C)}\right], \quad n \geq 1, \\
v_{0}=L^{-1}\left[\frac{T(0)}{(s-C)}\right] .
\end{gathered}
$$

Assuming $T(0)=C=1$, the successive approximate solutions $T_{n}=v_{0}+\cdots+v_{n}$ can be presented as

$$
\begin{aligned}
& T_{0}=L^{-1}\left[\frac{1}{(s-1)}\right]=e^{t} \\
& T_{1}=e^{t}-\varepsilon e^{2 t} \sinh (t)
\end{aligned}
$$

We can consider a Maple program for the approximation and set the truncated order as 7 and 12, respectively. The 7th term approximation and the 12th term approximation can be obtained as

$$
\begin{aligned}
& T_{7}=e^{t}-\varepsilon e^{2 t} \sinh (t)+\frac{3}{8} \varepsilon^{2}\left(-2 e^{3 t}+e^{5 t}+e^{t}\right)-\frac{5}{16} \varepsilon^{3}\left(3 e^{3 t}-3 e^{5 t}+e^{7 t}-e^{t}\right) \\
& +\frac{35}{128} \varepsilon^{4}\left(-4 e^{3 t}+6 e^{5 t}-4 e^{7 t}+e^{9 t}+e^{t}\right)-\frac{63}{256} \varepsilon^{5}\left(5 e^{3 t}-10 e^{5 t}+e^{11 t}+10 e^{7 t}-5 e^{9 t}-e^{t}\right) \\
& +\frac{231}{1024} \varepsilon^{6}\left(-6 e^{3 t}+15 e^{5 t}-6 e^{11 t}-20 e^{7 t}+e^{13 t}+15 e^{9 t}+e^{t}\right) \\
& -\frac{429}{2048} \varepsilon^{7}\left(7 e^{3 t}-21 e^{5 t}+21 e^{11 t}+35 e^{7 t}-7 e^{13 t}+e^{15 t}-35 e^{9 t}-e^{t}\right), \\
& T_{12}=e^{t}-\varepsilon e^{2 t} \sinh (t)+\frac{3}{8} \varepsilon^{2}\left(-2 e^{3 t}+e^{5 t}+e^{t}\right)+\cdots \\
& +\frac{676039}{4194304} \varepsilon^{12}\left(\begin{array}{c}
-12 e^{3 t}-220 e^{19 t}+66 e^{5 t}-792 e^{11 t}+e^{25 t} \\
-220 e^{7 t}-12 e^{23 t}+924 e^{13 t}+495 e^{17 t}-792 e^{15 t}+495 e^{9 t}+66 e^{21 t}+e^{t}
\end{array}\right) .
\end{aligned}
$$

Recall that (2) has an exact solution [13]

$$
T=\left[\frac{\varepsilon}{C}+\left(\frac{1}{T(0)^{2}}-\frac{\varepsilon}{C}\right) \exp (-2 C t)\right]^{-(1 / 2)}
$$

Setting $\varepsilon=0.00001$ in this paper, we apply the Padetechnique to the approximate solution $T_{12}$. In order to avoid the tediousness, the detail expression of the result is omitted here.

The approximate solutions from the ADOM and the LAPM are compared using the high iteration solutions $T_{\text {[LAPM] }}^{12 \text { th }}$ and $T_{[\mathrm{ADOM}]}^{12 \mathrm{th}}$ in Table 1 , respectively.

The exact solution (10), the approximate solutions $T_{[\mathrm{ADOM}]}^{12 \text { th }}, T_{[\mathrm{LAPM}]}^{12 \mathrm{th}}$, and the solution $T_{[\mathrm{LAM}]}^{12 \mathrm{th}}$ without the treatment using the Pade-technique are compared in Figure 1.

The results in Table 1 and Figure 1 illustrate that the LAPM has a higher accuracy, respectively.
Case II. For the coefficients $C=D=E=R_{h}=1$ and $\varepsilon=$ 0.00001 , (1) reduces to

$$
\begin{gathered}
\frac{d T}{d t}=T+h-0.00001 T^{3}, \\
\frac{d h}{d t}=-T-h .
\end{gathered}
$$

Setting the initial condition value $T(0)=h(0)=1$, we can derive the following iteration formula:

$$
\begin{gathered}
v_{n+1}(t)=-0.01 L^{-1}\left(\frac{1}{s-1} L\left[A_{n}\right]\right), \\
v_{0}(t)=T(0) L^{-1}\left(\frac{1}{s-1}\right)+h(0) L^{-1}\left[\frac{1}{s^{2}-1}\right] \\
=e^{t}+\sinh (t), \\
h_{n+1}(t)=-L^{-1}\left[\frac{L\left[v_{n}(t)\right]}{s+1}\right], \quad h_{0}=L^{-1}\left(\frac{h(0)}{s-1}\right)=e^{t} .
\end{gathered}
$$


TABLE 1: Comparisons between $T_{[\mathrm{ADOM}]}, T_{[\mathrm{LAPM}]}$, and the exact solution.

\begin{tabular}{lccc}
\hline$t$ & $T_{[\mathrm{ADOM}]}^{12 \mathrm{th}}$ & $T_{[\mathrm{LAPM}]}^{12 \mathrm{th}}$ & Exact solution \\
\hline 0.0 & 1.00000000000 & 1.00000000000 & 1.00000000000 \\
0.5 & 1.64870710600 & 1.64870710600 & 1.64870710600 \\
1.0 & 2.71819499800 & 2.71819499800 & 2.71819499700 \\
1.5 & 4.48126178100 & 4.48126146900 & 4.48126145500 \\
2.0 & 7.38709279800 & 7.38707674000 & 7.38707669500 \\
2.5 & 12.1738764100 & 12.1735250400 & 12.1735245800 \\
3.0 & 20.0498629300 & 20.0452439400 & 20.0452435100 \\
3.5 & 32.9785428200 & 32.9355166300 & 32.9355172400 \\
4.0 & 54.1154755000 & 53.8024166600 & 53.8023905200 \\
4.5 & 88.4548189000 & 86.5781681900 & 86.5781168700 \\
5.0 & 143.748571000 & 134.353636200 & 134.352939400 \\
\hline
\end{tabular}

where $h_{0}+\cdots+h_{n}$ is the $n$th approximation of $h(t)$. As a result, for $n=12$, we can obtain the approximate solution by means of the LAPM.

Define the residual functions $f(t)$ and $g(t)$ as

$$
\begin{gathered}
f=\left|\frac{d T}{d t}-T-h+0.01 T^{3}\right|, \\
g=\left|\frac{d h}{d t}+T+h\right| .
\end{gathered}
$$

The plotted functions $f(t)$ and $g(t)$ show that the iteration formula is reliable (Figure 2). Now we can analytically investigate the relationship between the temperature $T$ and the thermo-cline depth $h$, which is shown in Figure 3.

Remarks. This study only concentrates on the applications of the Adomian series in the linearization of the nonlinear equations. For various calculations of the Adomian series, readers are referred to the recent development of the method in $[3,4,14-16]$ and the applications in fractional different equations in [17-19]. It is interesting to point out that the results are the same as those of the one using the variational iteration method [20].

In the classical ADOM, the inverse operator should be used. For example, one can need to transform the differential equation

$$
\begin{aligned}
& \frac{d^{m} u}{d t^{m}}+R[u(t)]+N[u(t)]=g(t), \\
& u^{(k)}(0)=\frac{d^{k} u(0)}{d t^{k}}, \quad k=0, \ldots, m-1
\end{aligned}
$$

into the following equivalent integral equation

$$
\begin{gathered}
u=f(t)+\int_{0}^{t} \cdots \int_{0}^{t}(-R[u(s)]-N[u(s)]+g(s)) \underbrace{d s \cdots d s}_{m}, \\
u^{(k)}(0)=\frac{d^{k} u(0)}{d t^{k}}, \quad k=0, \ldots, m-1 .
\end{gathered}
$$

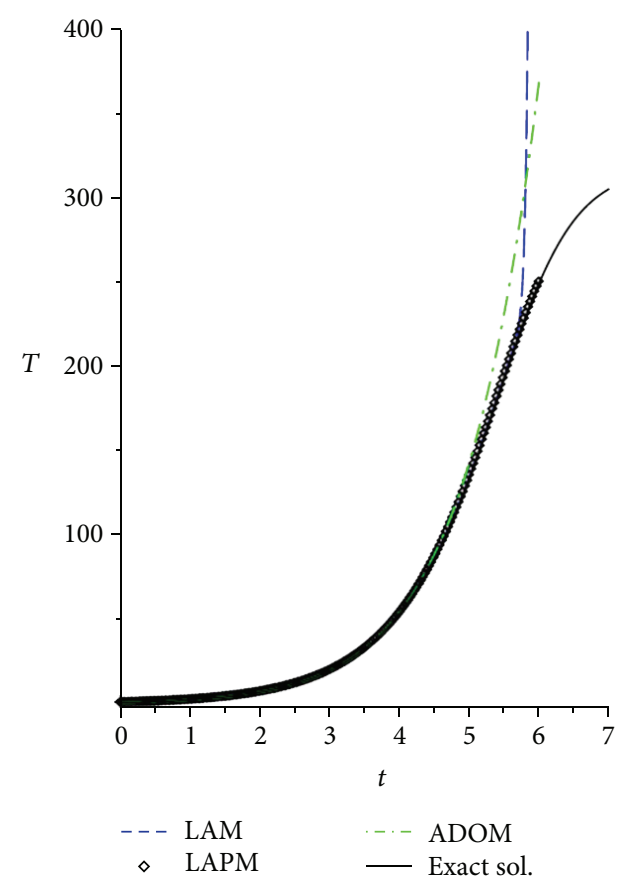

FIgURE 1: The comparisons of the approximate solutions using different methods.

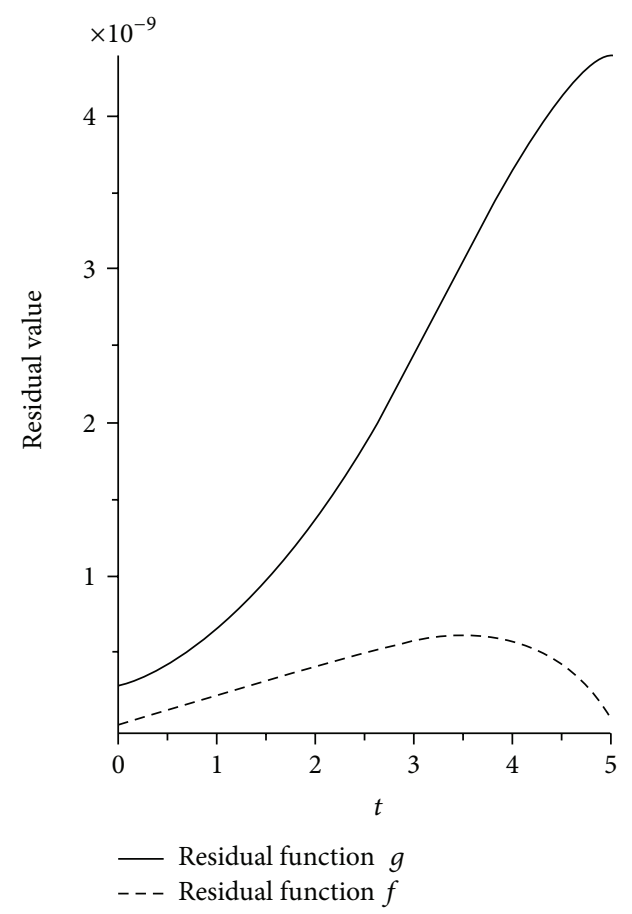

FIGURE 2: The residual functions: $f(t)$ and $g(t)$.

Here $\int_{0}^{t} \cdots \int_{0}^{t} \cdot \underbrace{d s \cdots d s}_{m}$ is called the inverse operator in the ADOM.

In Tsai and Chen's method, the solution procedure shows that the LAPM without using the inverse operator still keeps approximate solutions of higher accuracies. Furthermore, the initial iteration function can be readily determined. 


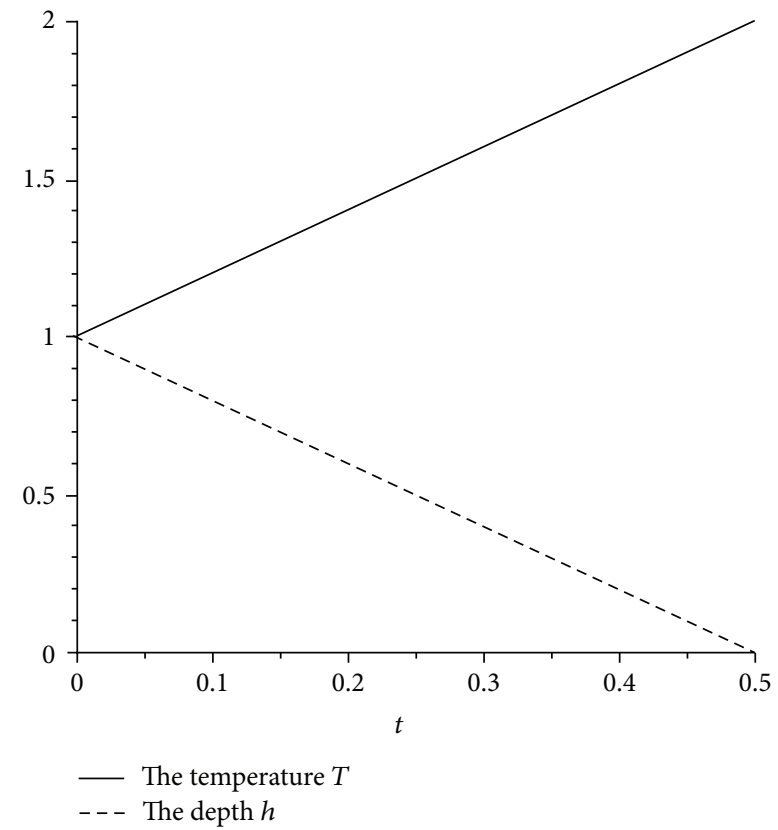

FIGURE 3: The temperature versus the thermo-cline depth $h$.

The method also can be extended to fractional differential equations [21] and $q$-difference equations.

\section{Conclusions}

With symbolic computation, the LAPM is used to approximately solve the ENSO model. We compared the approximate solutions with those from the ADOM and the LAPM, respectively. The results show that the LAPM has higher efficiency which can accelerate the convergence and enlarge the valid area of the approximate solution.

\section{Acknowledgment}

This work is supported by the Scientific Research Fund of Sichuan Provincial Education Department (12ZA085).

\section{References}

[1] G. Adomian, Solving Frontier Problems of Physics: The Decomposition Method, vol. 60 of Fundamental Theories of Physics, Kluwer Academic, Dodrecht, The Netherlands, 1994.

[2] H. Chu, Y. Zhao, and Y. Liu, "A MAPLE package of new ADM Padé approximate solution for nonlinear problems," Applied Mathematics and Computation, vol. 217, no. 17, pp. 7074-7091, 2011.

[3] H. Jafari and V. Daftardar-Gejji, "Revised Adomian decomposition method for solving a system of nonlinear equations," Applied Mathematics and Computation, vol. 175, no. 1, pp. 1-7, 2006.

[4] J.-S. Duan, "An efficient algorithm for the multivariable Adomian polynomials," Applied Mathematics and Computation, vol. 217, no. 6, pp. 2456-2467, 2010.
[5] C. Li, Z. Zhao, and Y. Chen, "Numerical approximation of nonlinear fractional differential equations with subdiffusion and superdiffusion," Computers \& Mathematics with Applications, vol. 62, no. 3, pp. 855-875, 2011.

[6] A.-M. Wazwaz, "A reliable modification of Adomian decomposition method," Applied Mathematics and Computation, vol. 102, no. 1, pp. 77-86, 1999.

[7] G.-C. Wu, "Adomian decomposition method for non-smooth initial value problems," Mathematical and Computer Modelling, vol. 54, no. 9-10, pp. 2104-2108, 2011.

[8] P.-Y. Tsai and C.-K. Chen, "An approximate analytic solution of the nonlinear Riccati differential equation," Journal of the Franklin Institute-Engineering and Applied Mathematics, vol. 347, no. 10, pp. 1850-1862, 2010.

[9] P.-Y. Tsai and C.-K. Chen, "Free vibration of the nonlinear pendulum using hybrid Laplace Adomian decomposition method," International Journal for Numerical Methods in Biomedical Engineering, vol. 27, no. 2, pp. 262-272, 2011.

[10] P.-Y. Tsai and C.-K. Chen, "A new algorithm on the solutions of forced convective heat transfer in a semi-infinite flat plate," Journal of Mechanics, vol. 27, no. 1, pp. 63-69, 2011.

[11] J.-Q. Mo and W.-T. Lin, "Generalized variation iteration solution of an atmosphere-ocean oscillator model for global climate," Journal of Systems Science \& Complexity, vol. 24, no. 2, pp. 271-276, 2011.

[12] R. Rach, "A convenient computational form for the Adomian polynomials," Journal of Mathematical Analysis and Applications, vol. 102, no. 2, pp. 415-419, 1984.

[13] J.-Q. Mo, W.-T. Lin, and J. Zhu, "The variational iteration solving method for El Niño/La Niño-southern oscillation model," Advances in Mathematics, vol. 35, no. 2, pp. 232-236, 2006.

[14] S. Saha Ray and R. K. Bera, "An approximate solution of a nonlinear fractional differential equation by Adomian decomposition method," Applied Mathematics and Computation, vol. 167, no. 1, pp. 561-571, 2005.

[15] V. Daftardar-Gejji and H. Jafari, "Adomian decomposition: a tool for solving a system of fractional differential equations," Journal of Mathematical Analysis and Applications, vol. 301, no. 2, pp. 508-518, 2005.

[16] H. Jafari and V. Daftardar-Gejji, "Solving linear and nonlinear fractional diffusion and wave equations by Adomian decomposition," Applied Mathematics and Computation, vol. 180, no. 2, pp. 488-497, 2006.

[17] S. Momani and Z. Odibat, "Analytical solution of a timefractional Navier-Stokes equation by Adomian decomposition method," Applied Mathematics and Computation, vol. 177, no. 2, pp. 488-494, 2006.

[18] S. Das and P. K. Gupta, "Approximate analytical solutions of time-space fractional diffusion equation by Adomian decomposition method and homotopy perturbation method," Communications in Fractional Calculus, vol. 2, no. 1, pp. 29-35, 2011.

[19] J. S. Duan, R. Rach, D. Baleanu, and A. M. Wazwaz, "A review of the Adomian decomposition method and its applications to fractional differential equations," Communications in Fractional Calculus, vol. 3, no. 2, pp. 73-99, 2012.

[20] G. C. Wu, "Challenge in the variational iteration method-a new approach to identification of the Lagrange mutipliers," Journal of King Saud University-Science, 2013.

[21] D. Q. Zeng and Y. M. Qin, "The Laplace-Adomian-Pade technique for the seepage flows with the Riemann-Liouville derivatives," Communications in Fractional Calculus, vol. 3, no. 1, pp. 26-29, 2012. 


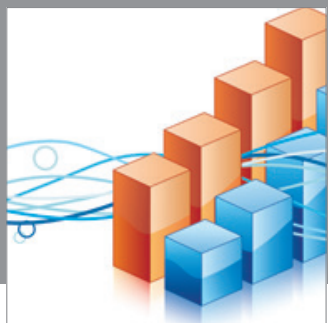

Advances in

Operations Research

mansans

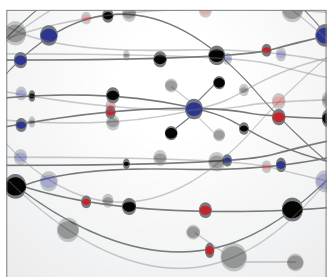

The Scientific World Journal
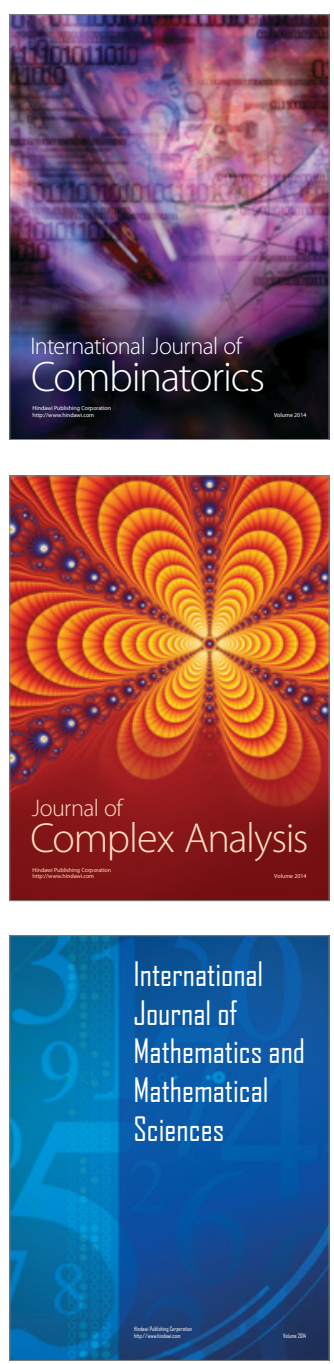
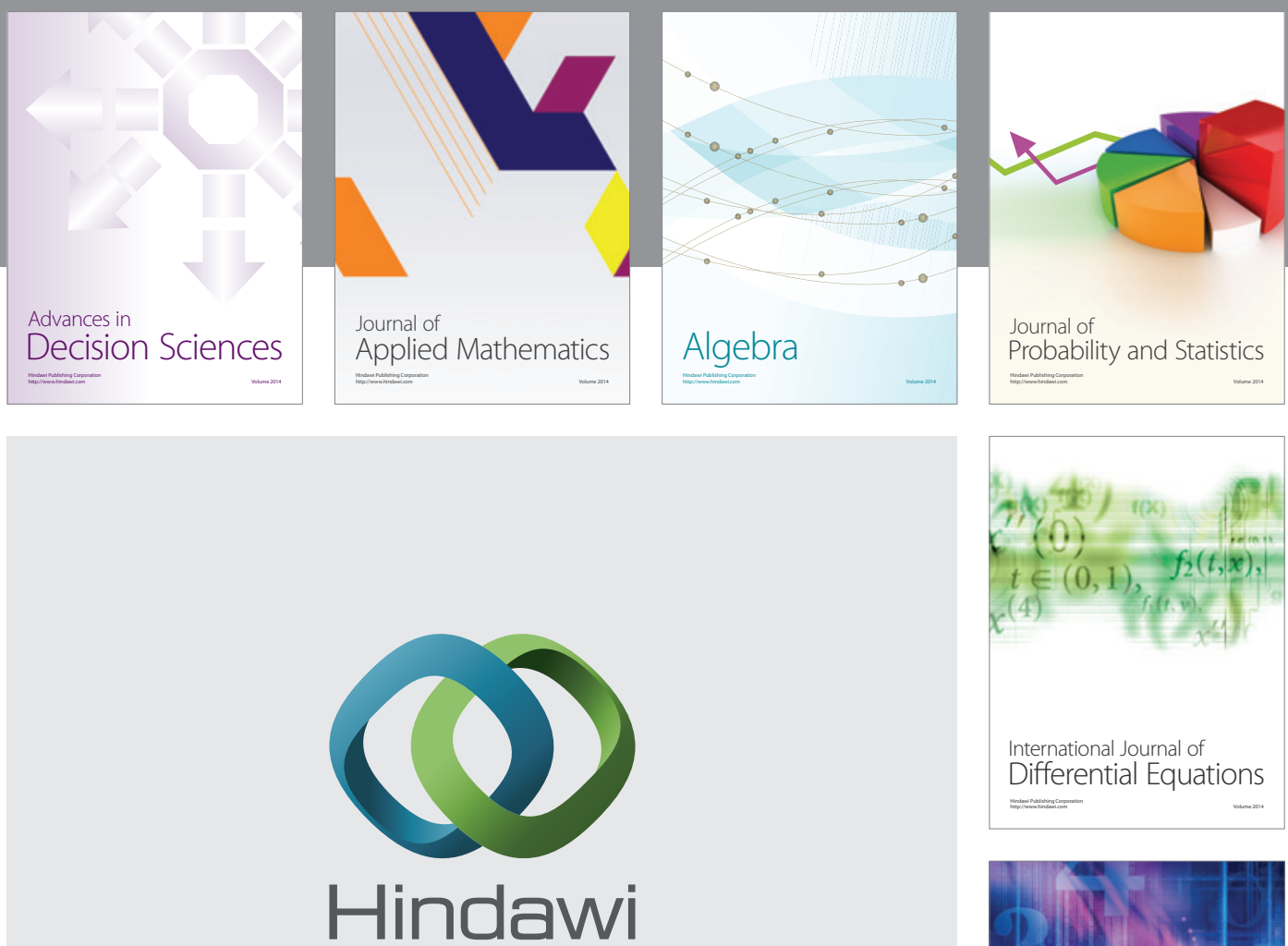

Submit your manuscripts at http://www.hindawi.com
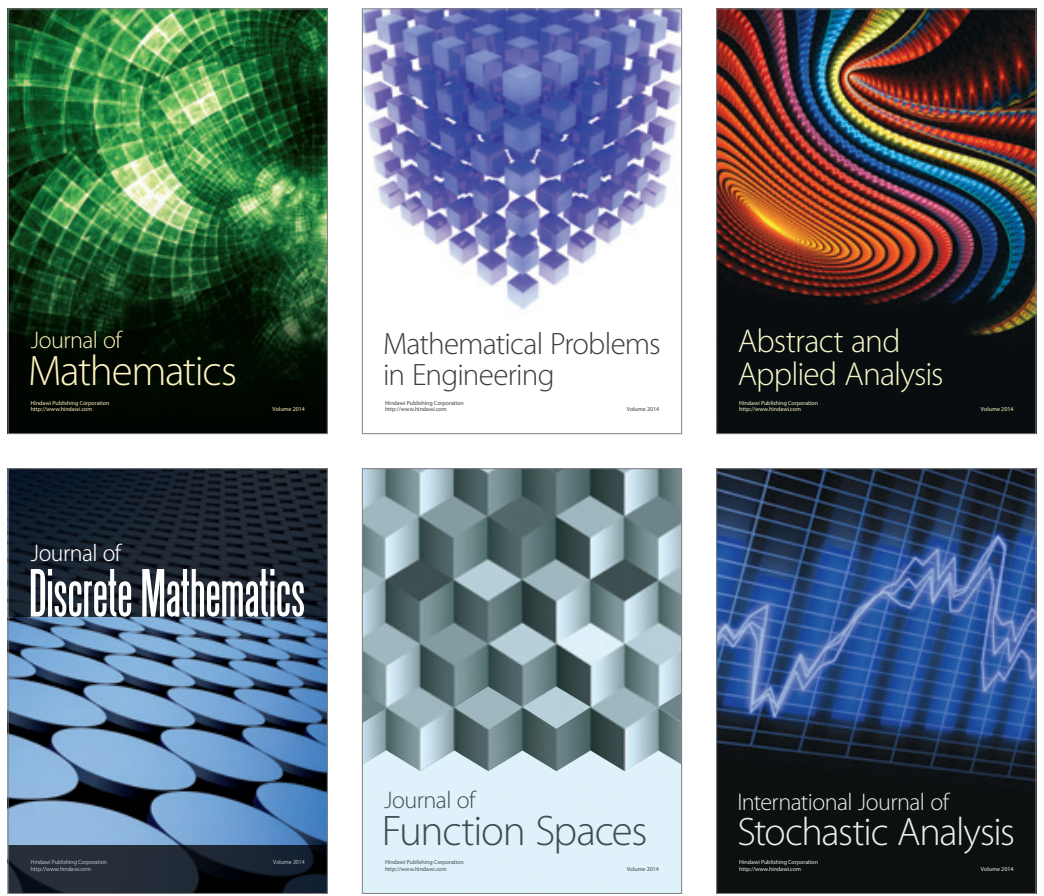

Journal of

Function Spaces

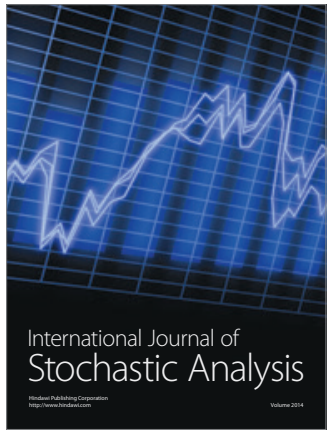

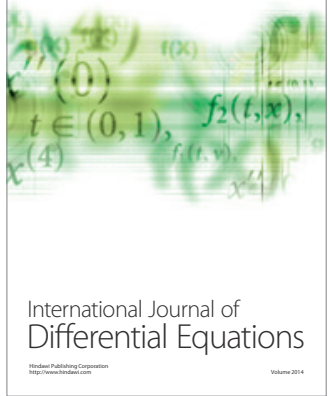
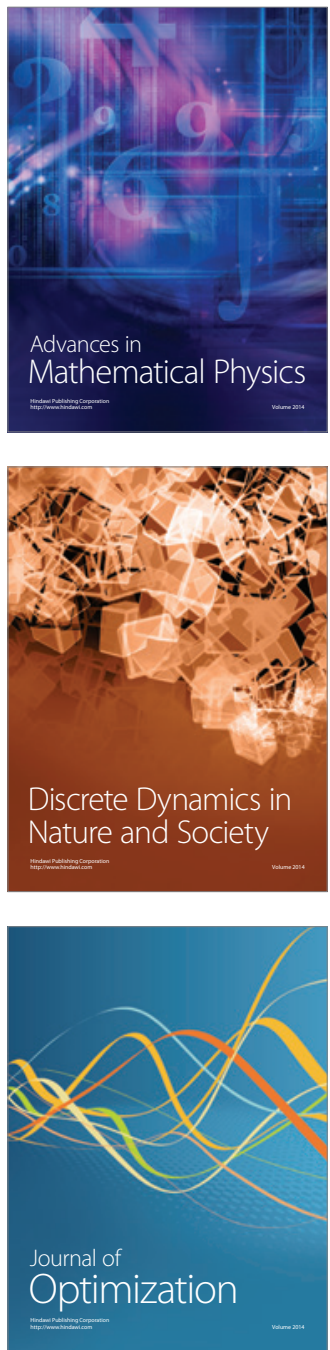